\title{
Events
}

\section{of Interest}

\section{June 2008}

Society For Vascular Surgery: 2008 Annual Meeting

Meeting: June 5-8, 2008, San Diego Convention Center, San Diego, California, For information, contact: Society for Vascular Surgery, 633 N. Saint Clair Street, 24th floor, Chicago, IL 60611 (telephone: +1 800 258-7188; E-mail: vascular@ vascularsociety.org).

34th Annual Toronto Thoracic Surgery Conference: June 6-7, 2008, Sutton Place Hotel, Toronto, Ontario, Canada. For more information, contact: Office of Continuing Education and Professional Development, Faculty of Medicine, University of Toronto (Telephone: 1-416-978-2719; Toll Free: 1-888-12-8173; Fax: 1-416946-7028; E-mail: help-SUR0807@ cmetoronto.ca; Conference Website: www. cme.utoronto.ca.

Innovations in Treatment of Cardiac Structural Disease: The Mediterranean Meeting

Meeting: June 6-7, 2008, Hilton Villa Igiea Palermo, Palermo, Sicily, Italy. For additional information, contact: Gina Esposito O'Malley, Project Specialist, Division of Cardiac Surgery, UPMC Presbyterian, The Heart, Lung and Esophageal Surgery Institute (telephone: +1-412-802-6591; fax: +1-412-648-6358; e-mail:espositogm@ upmc.edu).

other significant activities must be received at least 8 weeks before the desired month of publication. Information will be limited to title of meeting, date, place, and an address to obtain further information. Send announcements to Ryan Walther, Managing Editor, The Journal of Thoracic and Cardiovascular Surgery, 900 Cummings Center, Suite 221-U, Beverly, MA 01915 (E-mail: rwalther@prri.com).

16th European Conference on General Thoracic Surgery

Congress: June 8-11, 2008, Palazzo Re Enzo, Bologna, Italy. For more information, please contact: European Society of Thoracic Surgeons, PO Box 159, Exeter EX2 5SH, United Kingdom (telephone: +44 1392 430671; fax: +44 1392 430671; E-mail: sue@ests.org.uk; Website: http:// www.ests.org).
ISMICS 2008 Annual Scientific Meeting Meeting: June 11-14, 2008, Marriott Copley Place Hotel, Boston, Massachusetts. For further information see: www.ismics. org

Surgery-based Multimodality Therapy for Malignant Pleural Mesothelioma Meeting: June 13-14, 2008, Ritz Carlton Hotel, Dallas, Texas. For further information, contact Harvard Medical School Continuing Education Department (telephone: (617) 384-8600, Monday-Friday, 10 a.m. to 4 p.m. (EST); E-mail: hms-cme@hms. harvard.edu).

Western Thoracic Surgical Association 34th Annual Meeting

Meeting: June 25-28, 2008, Sheraton Keauhou Bay Resort \& Spa, Kona, HI USA. For information, contact: Western Thoracic Surgical Association, 900 Cummings Center Suite 221-U, Beverly, MA 01915 (telephone: 1-978-927-8330; fax: 1-978-5248890; E-mail: wtsa@prri.com).

Association of Physician Assistants in Cardiovascular Surgery 9th Annual Summer Educational Meeting Meeting: June 30-2 July 2, 2008, Keauhou Bay Hotel, Kailua Kona, Hawaii USA. For information, contact: APACVS, P. O. Box 4834, Englewood, CO 80155 (telephone: 1-877-221-5651; fax: 1-303-771-2550; www.apacv.net).

\section{July 2008}

International Academy of Cardiology: 14th World Congress on Heart Disease Annual Scientific Sessions, July 26-29, 2008, The Fairmont Royal York, Toronto, ON, Canada. For more information, contact: Asher Kimchi, M.D., Chairman, International Academy of Cardiology, PO Box 17659, Beverly Hills, CA 90209, USA (telephone: +1-310-657-8777; fax: +1-310659-4781; E-mail: klimedco@ucla.edu). 


\section{August 2008}

Scandinavian Association for Thoracic Surgery and Scandinavian Society for Extracorporeal Technology 57th Annual Meeting

The 57th Annual Meeting of Scandinavian Association for Thoracic Surgery and 28th Scandinavian Society for Extracorporeal Techonology will be held August 21-23, 2008 in Copenhagen, Denmark. Abstract submission deadline: May 5, 2008. For information, contact: ICS A/S, Strandvejen 169-171, P.O. Box 41, DK-2900 Hellerup, Denmark (telephone: +45-7023-7823; fax: +45 7023 7888; E-mail: sats2008@ics.dk; Website: www.scandinavianats.org).

\section{September 2008}

XI World Congress of International Society for Diseases of Esophagus (ISDE) Meeting: September 10-13, 2008, Novotel Budapest Congress Center, Budapest, Hungary. For information, contact: Peter Horvath, MD (telephone: +36-72536126; fax: +36 72536127; E-mail: Peter.O. Horvath@aok.pte.hu; Website: www. isdecongress2008.com).

Canadian Association of Thoracic Surgeons 11th Annual Meeting Meeting: September 11-14, 2008, World Trade and Convention Centre, Halifax, NS Canada. For imformation, contact: Joanne Clifton, MSc (telephone: +1-604-8755355; fax: +1 604-875-4036; E-mail: joanne.clifton@vch.ca; Website: www. canats.org).

\section{European Association for Cardio-} Thoracic Surgery 22nd Annual Meeting Meeting: September 13-17, 2008, Lisbon Congress Centre, Lisbon, Portugal. For information, contact: EACTS Executive Secretariat, 3 Park Street, Berkshire, SL4 1LU, UK (telephone: +44-1753-832166; fax: +44-1753-620407; E-mail: info@eacts.co. uk; Website: www.eacts.org).

\section{October 2008}

10th National Congress of the Turkish Cardiovascular Surgery Society Congress: October 17-21, 2008, Sheraton Hotel and Congress Center, Cesme Izmir Turkey, Abstract deadline: July 1, 2008. For information, contact: Interium Organization/United Expo (telephone: +90 212292 88 08/+90 23246500 85; fax: +90 212292 88 07/+90 23246500 86; E-mail: info@ interium.com.tr, serapaykutaka@gmail.com, info@tkdcd2008.org; Additional information: http://www.turkishcvs.org/).

\section{6th Triennial Brigham Cardiac Valve}

Symposium

Course: October 23-24, 2008, Fairmont Copley Plaza Hotel, Boston, MA. For information, contact: R. Morton Bolman, III, M.D., Brigham and Women's Hospital, 75 Francis Street, Boston, MA, 02115 (telephone: 1-617-732-6964; fax: 1-617-732-6559; E-mail: ljaffe1@ partners.org; Website: http://www. brighamandwomens.org/cardiacsurgery/events. aspx).

\section{CHEST 2008}

Annual Meeting: October 25-30, 2008, Philadelphia, Pennsylvania. For more information contact: ACCP Customer Relations (telephone: 847-498-8387; E-mail: accp@ chestnet.org).

\section{November 2008}

Southern Thoracic Surgical Association 55th Annual Meeting

Meeting: November 6-8, 2008, Los Pines Resort, Austin, Texas. For information, contact: Nancy Gray Puckett, 633 North St. Clair, Suite 2320, Chicago, IL 60611 (telephone: +1-800-685-7872; fax: +1312-202-5801; E-mail: stsa@stsa.org; Website: www.stsa.org).

\section{June 2009}

Fifth Biennial Meeting of The Society for Heart Valve Disease

Meeting: June 2009, Ritz Carlton Hotel, Berlin Germany. For information, contact: SHVD Executive Secretariat, Heart Science Centre, Harefield Hospital, Harefield Middlesex, UB9 6JH, UK (telephone: +44 (0)1895-828977; Fax: +44 (0)1895828902; Email: secretariat@shvd.org; Website: www.shvd.org). 